\title{
Effects of Stereotype and Social Distance toward Foreign Students on Multicultural Acceptance of Social Welfare Students
}

\author{
So Yun $\mathrm{Choi}^{1}$ \\ ${ }^{I}$ Associate Professor, Department of Social Welfare, Namseoul University, Korea, sychoi@nsu.ac.kr
}

\begin{abstract}
In a multicultural Korean society, social welfare professionals have ethical responsibilities to equip themselves with cultural competence and provide services to diverse clients without discrimination and prejudice. Therefore, this study examined the level of multicultural acceptance of social welfare students as a prerequisite competence for multicultural social welfare practice and identified the effects of social welfare students' stereotypes and social distance toward foreign students on multicultural acceptance. From October 1 to 15, 2019, a survey was conducted targeting first to fourth year students majoring in social welfare at a university located in Chungcheongnam-do. Using data collected from 134 students (female 53\%, male 47\%), this study identified the effects of antecedent variables on multicultural acceptance through a hierarchical regression analysis. The average of multicultural acceptance was 3.8, with 3.3 stereotype, and 3.1 social distance. The average multicultural acceptance of first-year students was relatively higher than that of senior students. As a result of the hierarchical regression analysis, the influence of grade was statistically significant in Model 1 and Model 2, but Models 1 and 2 did not have statistically significant explanatory power. In Model 3, it has been identified that the frequency of contact with foreign students, stereotype, and social distance affected multicultural acceptance at $\mathrm{p}<.001$ level with an adjusted $\mathrm{R}^{2}$ of .261. In order to foster academic training of social welfare students for effective multicultural social welfare practices, a curriculum related to multicultural acceptance should be provided in the undergraduate courses.
\end{abstract}

Keywords: Multicultural Acceptance, Social Distance, Stereotype, Social Welfare Student

\section{Introduction}

\subsection{Purpose of the Study}

Social workers must accept clients as they are. In particular, it is necessary to respect the diversity of each client, such as race, ethnicity, gender, and culture, and to have cultural competence to utilize the strength of the client's unique culture in the process of social welfare practice.

Due to the increased transnational movement, various races, and ethnic groups, including married immigrants and foreign workers, have flowed into Korea since the mid-2000s, and Korea has entered a multicultural society without preparation. Multicultural policies for migrants were presented, and cultural competence was emphasized as essential knowledge and skills for social workers in multicultural practices.

Efforts to respond to these social changes are not limited to social welfare practices and policies. To train practitioners prepared for a multicultural society, the Korea Council for Social Welfare Education has included 'social welfare and cultural diversity' in its standard curriculum to foster human resources

Received: June 16, 2021; $1^{\text {st }}$ Review Result: August 01, 2021; $2^{\text {nd }}$ Review Result: September 17, 2021 Accepted: October 31, 2021 
suitable for the changed social environment.

Although racial and ethnic diversity coexisted in Korea from the past, it was in the mid-2000s that a full-fledged discussion on the multicultural phenomenon of society began. In the meantime, it was not an easy task for Korea to accept migrants with racial and ethnic differences as members of society, because mono-culture and mono-ethnic sentiment prevailed.

The deep-rooted mono-ethnic and mono-cultural sentiments of Korean society are also related to discriminatory stereotypes and social distance toward multicultural migrants. Through in-depth interviews with eight people in their 20s, Kim H.[1] examined stereotypes of migrants according to their country of origin and confirmed that they have different stereotypes about Anglo-Americans, Asians, Korean-Chinese, and that these stereotypes tend to lead to discriminatory attitudes. In addition, Kim K.[2] found that the social distance among students increased in the order of Asians, whites, blacks, and Arabs in a study on social distance among nursing college students.

In order to prevent and alleviate various social problems such as discrimination, prejudice, and exclusion, caused by the entry into a multicultural society, the government is supporting social integration of migrants by presenting multicultural policy directions in cooperation with various related ministries. However, there are concerns that the government's directions rather reversely discriminate against non-multicultural people, and there are criticisms that its policy stance does not reflect the value of inclusiveness in a multicultural society[3].

Changes in social environment due to the influx of migrants are the same in universities. The number of foreign students in Korea is estimated to be 160,000 in 2019 before COVID-19[4]. These foreign students are distributed at universities across the country, and Korean university students experience ethnic and racial diversity on campus, so embracing and coexisting in a multicultural society is needed throughout Korea.

The first thing that is required to become a multicultural society is the acceptance of multiculturalism, which accepts differences as they are. In particular, in the case of social welfare professionals who provide services to people, "acceptance" that means "to accept clients as they are" is the essence of professional ethics. Multicultural acceptance of immigrants with various cultural backgrounds is an essential element that helping professionals must possess in a multicultural society.

However, it is not always easy to accept the cultural differences of migrants due to the stereotypes and social distance people have toward different race and ethnicity because of deep-seated mono-ethnic sentiment. In particular, since Korea has traditionally adhered to pure bloodism and monoculture, there is a tendency to show a sense of distance toward international students with multiracial and multicultural backgrounds from stereotypes or discriminatory attitudes.

As a prerequisite for effectively performing multicultural social welfare practice in a multicultural Korean society, this study examined the level of multicultural acceptance of social welfare students who must play a pivotal role in multicultural social welfare practices. In addition, this study is intended to help find implications and directions for fostering academic training of social welfare students for multicultural social welfare practices. Therefore, this study tried to identify the effects of stereotype and social distance toward rapidly increasing foreign students on the multicultural acceptance of social welfare students.

\subsection{Research Questions}

Specifically, the following research questions are to be identified as the structure of this study.

First, what is the level of stereotype and social distance toward foreign students among social welfare students?

Second, does the frequency of contact with foreign students affect social welfare students' multicultural acceptance? 
Third, do social welfare students' stereotype and social distance toward foreign students affect their multicultural acceptance?

\section{Theoretical Background}

\subsection{Multicultural Acceptance}

Multicultural acceptability is an attitude that accepts the changes caused by the entry of a multicultural society as positive and supports the social value in which various ethnic groups or races coexist[5]. Currently, Korea is a multicultural society where various races and ethnicities coexist, but discrimination and prejudice against migrants still exist. Due to Korea's deep-rooted sentiment of pure bloodism and homogeneous ethnicity, there is an exclusionary attitude toward multiracial and multicultural immigrants. According to the 2018 Korean Multicultural Acceptance Survey, conducted every three years from 2012, the willingness to exchange with multicultural people and expectations for assimilation have increased compared to 2015, requiring national efforts to enhance multicultural acceptance[6].

Since the inclusion of diversity is essential in a multicultural society, multicultural acceptance that accepts ethnically and racially diverse clients as they are is essential. In particular, in the case of social workers who practice according to ethical guidelines that must accept the unique characteristics of their clients as they are, accepting the unique culture and values of migrants from multicultural societies is a basic premise for social welfare practices.

\subsection{Stereotype}

A stereotype is the notion that attributes an individual's characteristics to a limited category simply because the individual belongs to a specific group category without considering or acknowledging the individual's individuality or ability[7]. Due to stereotypes, the unique characteristics of an individual are overlooked or not respected, and errors occur that are understood and interpreted by the values of the mainstream group.

In particular, negative stereotypes act as triggers of discrimination or prejudice. Because they are expressed as discriminatory behaviors, stereotypes toward foreign students can cause a sense of social distance or inability to accept differences[8].

\subsection{Social Distance}

Social distance, as opposed to social intimacy, refers to an individual's attitude toward an individual or group with a specific status. Social distance means the degree to which one can become friendly through interpersonal interactions[9]. The studies of social distance are predominantly conducted on a minority group that is heterogeneous from the mainstream group. The minority groups that are not welcomed by the mainstream group include North Korean defectors, foreign workers, young people from multicultural families, the elderly with dementia, the disabled, AIDS patients, and migrants.

\subsection{Studies on Antecedent Factors Affecting Multicultural Acceptance}

With the rapid influx of migrants into Korean society since the mid-2000s, social integration for the settlement of migrants has become a major social issue. It is a great task for a multicultural society to accept and embrace multi-ethnic immigrants, such as foreign workers, marriage immigrant women, and foreign students.

Since multicultural acceptance research mainly focus on the degree of acceptance of a particular subject of multiculturalism, most of them are conducted on human service workers or on children, 
teenagers or college students who may have a possibility of contact with multiculturalism. The multicultural acceptance in previous studies is analyzed as a dependent variable or an independent variable. As an independent variable, multicultural acceptance is linked to organizational effectiveness. However, most of the studies set multicultural acceptance as a dependent variable.

Demographic and sociological characteristics such as gender are considered as antecedent variables affecting multicultural acceptance, but these mainly have the meaning of control variables. Multicultural contact experiences such as related educational experience and volunteer experience are suggested as antecedent variables affecting multicultural acceptance, and it is confirmed that people with experience in multicultural education have higher multicultural competence and multicultural acceptance than those who do not. In addition, it is verified that social intimacy toward foreigners have the mediating effect in the impact of cultural sensitivity on multicultural acceptance[10-12].

\section{Research Method}

\subsection{Sampling and Research Subjects}

From October 1 to 15, 2019, a survey was conducted targeting first to fourth grade students majoring in social welfare at a university located in Chungcheongnam-do. A total of 150 questionnaires were distributed, and data collected from 134 people (female 53\%, male 47\%) were used.

The subjects of this study agreed to its purpose of this study and voluntarily participated in the survey. During the break of the lectures in grades 1-4, the researcher explained the purpose of the survey and conducted it. For a total of 250 students enrolled in the Department of Social Welfare, the convenience sampling method was used for sampling.

\subsection{Ethical Considerations}

The researcher informed the participants about the purpose, necessity, and use of the research results, and explained the confidentiality of the research and the right to refuse the investigation. They were also notified that there were no disadvantages in case of non-participation. Participants voluntarily participated in the research process without any coercion.

\subsection{Questionnaire Composition and Scale}

As for general characteristics, the questionnaire included content such as gender, grade, and age, and consisted of questions asking about international students in universities and their personal experiences related to multiculturalism. For multicultural acceptance, the original items of the measure used by Park H. in a multicultural acceptance scale validation study for college students were utilized[13].

This five-point Likert scale consists of three sub-concepts namely cognitive, affective, and behavioral tendencies. A Total of 37 items include 13 items for cognitive acceptance, 12 items for affective acceptance, and 12 items for behavioral tendency. Multicultural acceptance is a five-point Likert scale, and a higher score is interpreted as a higher degree of acceptance. Cronbach's Alpha was used to measure the reliability of the scale. In this study, the reliability was .917 .

Cognitive acceptance consists of 13 items, such as "I think it is important to understand other cultures," "I think people's viewpoints may be different from mine," and "I think our people are the best in the world (reverse question)." Affective acceptance consists of 12 items, such as "I feel uncomfortable about racism in our society," "I feel that people with different cultural backgrounds may have different values," "I feel more likable to people from a certain country because of their skin color (reverse question)." Behavioral tendency consists of 12 items, such as "I get along well with people from different cultural 
backgrounds" and "I accept foreign students as equal students as me."

Stereotypes were measured with 11 pairs of adjectives developed by Corrigan and Schudmidt[14] and corrected and supplemented by Park S.[15]. On a five-point scale, the higher the score, the greater the positivity toward the subject. It is a measure of the degree of thinking about foreign students. It is composed of adjectives such as "humble," "dirty"(reverse question), "kind," "lazy" (reverse question), "stubborn" (reverse question), and "reasonable." On a five-point scale, the higher the score, the greater the positivity toward the subject (less stereotypes). In this study, the reliability was .804 .

The social distance is a six-item five-point scale used by Kim and Oh[16] and Park S.[15]. The higher the score, the greater the social distance. The questions were whether it was acceptable as a neighbor, workplace, family, spouse, and date. The reliability was .886 in this study.

\subsection{Analysis Method}

For a statistical analysis, SPSS 23.0 was used. For the analysis of general characteristics of respondents, descriptive statistics were used, and the t-test was used for the analysis of difference in multicultural acceptance between the lower and upper grades. The effects of frequency of contact with foreign students, stereotypes, and social distance on multicultural acceptance was analyzed by hierarchical regression analysis.

\section{Research Result}

\subsection{General Characteristics of Respondents}

The respondents were 134 social welfare students, with 63 men (47\%) and 71 women (53\%) showing a rather high female response rate. The respondents' grades were 26 each in the first and second grades, 38 in the third grade, and 44 in the fourth grade. The average age was 21.26 years.

[Table 1] General Characteristics of Respondents

\begin{tabular}{|c|c|c|c|c|c|c|c|}
\hline \multicolumn{2}{|c|}{ Gender } & \multicolumn{4}{c|}{ Grade } & \multicolumn{2}{c|}{ Age } \\
\hline Male & Female & 1 & 2 & 3 & 4 & Minimum & 18 \\
\hline 63 & 71 & 26 & 26 & $\begin{array}{c}38 \\
(19.4 \%)\end{array}$ & $\left.\begin{array}{c}428.4 \%) \\
(32.8 \%)\end{array}\right)$ & Maximum & 26 \\
\cline { 5 - 8 } & $(53 \%)$ & $(19.4 \%)$ & Average & 21.26 \\
\hline
\end{tabular}

\subsection{Frequency of Contact with Foreign Students}

As a result of checking the average number of contact with foreign students at school per week, as shown in [Table 2], the frequency of contact one or two times a week was $73(54.5 \%)$, and three or four times was $25(18.7 \%)$. The case of contact more than five times was 20 (14.9\%). The number of students who did not make any contact at all was 16 , or $11.9 \%$.

[Table 2] Frequency of Contact with Foreign Students

\begin{tabular}{|c|c|c|c|}
\hline $\begin{array}{c}\text { Contact } \\
\text { Frequency }\end{array}$ & Frequency & \% & Accumulation \% \\
\hline 0 & 16 & 11.9 & 11.9 \\
$1-2$ & 73 & 54.5 & 66.4 \\
\hline $3-4$ & 25 & 18.7 & 85.1 \\
\hline
\end{tabular}




\begin{tabular}{|l|c|c|c|}
\hline $\begin{array}{l}\text { More than 5 } \\
\text { Total }\end{array}$ & 20 & 14.9 & 100.0 \\
\hline
\end{tabular}

\subsection{Descriptive Statistics of Key Variables}

The social welfare students had a positive perception toward foreign students with an average stereotype score of 3.26, but a rather low score with an average social distance of 3.1.

The average of multicultural acceptance was 3.79, which was higher than the average of the other two variables. As for the average score of multicultural acceptance by grade, the first grade showed the highest acceptance with 4.0 and the third grade showed the lowest acceptance with 3.7.

[Table 3] Descriptive Statistics of Key Variables

\begin{tabular}{|c|c|c|c|c|c|}
\hline & $\mathbf{N}$ & Minimum & Maximum & Mean & Std. Deviation \\
\hline Stereotype & 134 & 2.55 & 5.00 & 3.2619 & .38971 \\
\hline Social Distance & 134 & 1.00 & 4.67 & 3.1045 & .70227 \\
\hline $\begin{array}{c}\text { Multicultural } \\
\text { Acceptance }\end{array}$ & 134 & 3.00 & 4.87 & 3.7938 & .40768 \\
\hline
\end{tabular}

[Table 4] Multicultural Acceptance by Grade

\begin{tabular}{|c|c|c|c|c|}
\hline Grade & $\mathbf{N}$ & Mean & Std. Deviation & Std. Error Mean \\
\hline 1 & 26 & 4.0028 & .39063 & .07661 \\
\hline 2 & 26 & 3.7688 & .37217 & .07299 \\
\hline 3 & 38 & 3.7007 & .38361 & .06223 \\
4 & 44 & 3.7654 & .42770 & .06448 \\
\hline
\end{tabular}

\subsection{Results of Hierarchical Regression Analysis}

The effect of antecedent variables on multicultural acceptance was confirmed through hierarchical regression analysis. Model 1 added gender and grade, Model 2 added frequency of contact with foreign students to Model 1, and Model 3 added social distance and stereotype to confirm the influence of antecedent variables on multicultural acceptance of social welfare students.

The multicollinearity of the regression analysis was confirmed by the tolerance and variance inflation factor. In the case of tolerance, the requirement of $>.01$ was satisfied with $.814 \sim .100$, and the VIF (variance inflation factor) of 1.00 1.27 met the requirement of $<10$, indicating that there was no problem of multicollinearity.

As a result of the study [Table5], Model 1 showed that the lower grades had high multicultural acceptance, but the model was not statistically significant. The frequency of contact with foreign students in Model 2 was not significant. Due to the effect of grade, Model 2 was statistically significant at the $\mathrm{p}<.05$ level, but the amount of change in the $\mathrm{F}$ value had no statistical significance.

As a result of analysis by adding stereotype and social distance variables to model 2 , it was found that model 3 explains multicultural acceptance with an adjusted regression coefficient of .216 at the significance level $\mathrm{p}<.001$. Frequency of contact with foreign student, stereotype, and social distance variables all influenced multicultural acceptance.

The amount of change in $\mathrm{R}^{2}$ from Model 2 to Model 3 (Model $3 \mathrm{R}^{2}$ - Model $2 \mathrm{R}^{2}$ ) was .182, and the 
amount of change was statistically significant at the level of .001. In other words, the higher the frequency of contact, the fewer the stereotypes, the less the social distance, the higher the multicultural acceptance.

[Table 5] The Result of Hierarchical Regression

\begin{tabular}{|c|c|c|c|c|c|c|}
\hline & \multicolumn{2}{|c|}{ Model 1} & \multicolumn{2}{|c|}{ Model 2} & \multicolumn{2}{|c|}{ Model 3} \\
\hline & $\beta$ & $\mathbf{t}$ & $\beta$ & $\mathbf{t}$ & $\beta$ & $\mathbf{t}$ \\
\hline Gender $($ Female $=0)$ & -.025 & -.359 & -.038 & -.554 & .003 & .053 \\
\hline Grade & -.069 & $-2.21 *$ & -.064 & $-2.04 *$ & -.023 & -.744 \\
\hline Frequency of Contact & & & .057 & 1.889 & .076 & $2.104 *$ \\
\hline Stereotype & & & & & .324 & $3.572 * * *$ \\
\hline Social Distance & & & & & .155 & $-3.359 * * *$ \\
\hline Constant & \multicolumn{2}{|c|}{3.996} & \multicolumn{2}{|c|}{3.81} & \multicolumn{2}{|c|}{2.14} \\
\hline $\mathrm{R}^{2}$ & \multicolumn{2}{|c|}{.037} & \multicolumn{2}{|c|}{.063} & \multicolumn{2}{|c|}{.245} \\
\hline Adjusted $\mathrm{R}^{2}$ & \multicolumn{2}{|c|}{.022} & \multicolumn{2}{|c|}{.041} & \multicolumn{2}{|c|}{.216} \\
\hline$\triangle \mathrm{R}^{2}$ & \multicolumn{2}{|c|}{.037} & \multicolumn{2}{|c|}{.026} & \multicolumn{2}{|c|}{.182} \\
\hline F Value & \multicolumn{2}{|c|}{2.51} & \multicolumn{2}{|c|}{2.90} & \multicolumn{2}{|c|}{8.31} \\
\hline F Sig. & \multicolumn{2}{|c|}{.085} & \multicolumn{2}{|c|}{.038} & \multicolumn{2}{|c|}{.000} \\
\hline Sig. F Change & \multicolumn{2}{|c|}{.085} & \multicolumn{2}{|c|}{.061} & \multicolumn{2}{|c|}{.000} \\
\hline
\end{tabular}

$* \mathrm{p}<.05, * * \mathrm{p}<.01, * * * \mathrm{p}<.001$

\section{Discussion: Implications of the Findings}

This study examined the level of multicultural acceptance of social welfare students, who are prospective social workers who will play a pivotal role in multicultural social welfare practices and identified the effect of antecedent variables on multicultural acceptance.

According to the findings of this study, the average of multicultural acceptance was 3.79, indicating a relatively high average. However, there were some differences in multicultural acceptance depending on the grade level, and it was found that the multicultural acceptance of the first and second grade students was higher than the average of the third and fourth grade students.

The multicultural acceptance of higher grades was lower than that of lower grades, even though they had more contact with foreign students during lectures than the lower grades. This is because not only the frequency of contact but also the quality of contact is a prerequisite for multicultural acceptance.

Nam and Hong[17] confirmed that close contact with foreign immigrants has a relatively greater effect on multicultural acceptance than superficial contact. Therefore, it is important to expand multicultural acceptance through interchangeable activities with foreign students as well as contact during lectures.

The average of stereotype was 3.26, which was more positive than the average, and the average of social distance showed a rather low score with 3.1. This was 1.71 in a study by Noh and Lee[18], who studied the social distance of nursing students. That was more positive than in this study because the higher the score, the higher the degree of social distance.

In Model 1 of the regression analysis, grade level affected multicultural acceptance, but the model was not statistically significant. Model 2 was statistically significant and grade level affected multicultural acceptability, but the amount of change in the F value between Model 1 and Model 2 was 
not significant.

Unlike previous studies, the frequency of contact did not have a statistically significant effect on multicultural acceptance in Model 2. This result is different from previous studies that showed that the direct and indirect contact experience of multiculturalism affects multicultural acceptance[19]. The results indicate that the frequency of contact through lectures at school does not have a large effect on actual multicultural acceptance.

In Model 3, it was confirmed that social welfare students' stereotypes and social distance toward foreign students affect multicultural acceptance. In other words, the more negative stereotypes there were, the lower the multicultural acceptance, and the greater the social distance, the lower the multicultural acceptance.

The same results can be confirmed in the previous studies that social intimacy of social welfare college students affects multicultural acceptance[12], and that social distance of nursing students affects multicultural acceptance[18]. In addition, it can be confirmed in the study of Shim and Lee[20] that multicultural stereotypes and multicultural experiences of adolescents affect multicultural acceptance.

Korea tends to exclude immigrants due to its deep-rooted pure-bloodism and monoculture, and it can be seen that social welfare students are no exception from the dominant values of this society. Since social workers meet a variety of clients in the field, they must have cultural competence to accept clients as they are and to utilize the strengths of their own culture in practice. However, as stereotypes and sense of distance toward immigrants, including foreign students, are in violation of the Code of Ethics for Social Workers, a curriculum should be organized to promote the cultural awareness of social welfare students.

\section{Conclusion}

Suggestions based on the results of this study are as follows. For effective multicultural social welfare practices, social welfare students must embrace racial and ethnic diversity. To this end, various activities to enhance multicultural receptive should be provided in the undergraduate courses. Above all, in order to effectively support clients with cultural diversity, the "social welfare and cultural diversity," which is presented in the standard curriculum guidelines of the Korean Council for Social Welfare Education, must be organized in the curriculum. The American Council for Social Welfare Education recommends detailed guidelines for acquiring multicultural competence in the undergraduate courses. This is because multicultural phenomenon is a global trend, and cultural competence is essential to effectively practice it for various clients. Beyond the guidelines provided in the standard curriculum of the Korean Council for Social Welfare Education, it should be organized into the curriculum of each university so that students can complete it.

The experience of contact with various cultures should be promoted. Although frequency of contact with foreign students does not have a significant effect on multicultural acceptance in this study, prior studies have also discussed multicultural experience as a leading variable affecting multicultural acceptance. In the undergraduate course, it is necessary to increase the understanding of multicultural clients and to accommodate diversity through the expansion of volunteer work and educational opportunities to access multicultural clients.

It is possible to expand the experience of exchanges with foreign students. Furthermore, it is necessary to provide opportunities for formalized activities with foreign students from various cultural backgrounds in the university to reduce social distance toward migrants and to help students grow as social workers prepared for multicultural society.

Regarding multicultural acceptance, this study has limitations because it does not target various foreign immigrants, but only foreign students. In Korea's multicultural society, multicultural acceptance has to include various races and ethnicities, so there should be follow-up studies that expand the scope 
of future research.

In addition, there is a limitation in examining only the influence of antecedent variables affecting multicultural acceptance through random sampling. In future research, it will be necessary to confirm the effects of multicultural acceptance on various dependent variables through follow-up studies.

\section{References}

[1] Kim Heejeong, Exploring Stereotypes according to the Nationality of Immigrants living in Korea, Media, Gender \& Culture, (2017), Vol.32, No.2, pp.125-174. DOI : 10.38196/mgc.2017.06.32.2.125

[2] Kim Kyoungsook, Factors Affecting of Social Distance Toward Multiracial Society in Nursing Students, Journal of The Korean Data Analysis Society, (2012), Vol.14, No.4, pp.2039-2051. UCI : G704-000930.2012.14.4.044

[3] Lee Eunkyoung, Hwang Hyunsook, Multicultural Policy Research Report: Focusing on new search for and voice of field, The Hope Institute, (2016)

[4]Current status graph of foreign students (universities) in Korea, Retrieved in e-nara index http://www.index.go.kr/potal/main/EachDtlPageDetail.do?idx_cd=1534.

[5] Yun Injin, Song Youngho, South Koreans' Perceptions of National Identity and Acceptance of Multiculturalism, The Korean Journal of Unification Affairs, (2011), Vol.23, No.1, pp.143-192. UCI : G704-000548.2011.23.1.008

[6] 2018 National Multicultural Receptivity Survey, Seoul: Ministry of Gender Equality and Family, (2018)

[7] Kim Hyunah, Lee Jayoung, The Effect of Multi-cultural Counselor Education on Counselor' Prejudice and Multicultural Counseling Self-efficacy through E-learning, Multiculture and Peace, (2018), Vol.12, No.1, pp.59-79. DOI : 10.22446/mnpisk.2018.12.1.003

[8] Seo Boram, Sim Imsook, Kim Hyoil, A Study on the Influence of National Identity upon the Reciprocity Willingness of Adolescents: With a Focus on Mediating Effects of Stereotypes and Discrimination, Multiculture and Peace, (2019), Vol.13, No.2, pp.44-65. DOI : 10.22446/mnpisk.2019.13.2.003

[9] Kim Doori, Lee Seoyoung, Lee Songhee, Lee Yejoo, Lee Youngjae, Lee Wonkyoung, Lee Yoojeong, Factors Affecting Social Distance between Nursing Students and Older Adults with Dementia: Focusing on Dementia Knowledge, Attitude and Ageism, Journal of the Korea Convergence Society, (2019), Vol.10, No.7, pp.373-381. DOI : 10.15207/JKCS.2019.10.7.373

[10] Jee Sul Hyun, Lim Anna, A Study on the Effects of the Social Capital of Multi-cultural Social Welfare Practitioners on the Multi-cultural Acceptance, Global Culture Review, (2017), Vol.8, No.2, pp.1-21

[11] Kim Yu Ri, Relations between Multicultural Education and Multicultural Capabilities: Mediated Effect of Multicultural Receptivity by the Workers Engaged in the Dream-start Project, Graduate School of Sookmyoung Womans University, Master's thesis, (2020)

[12] Kim Kyounho, Effects of intercultural sensitivity on multicultural acceptance among social work students: Mediating effect of social familiarity to foreigners, Korean Journal of Social Welfare Education, (2013), Vol.22, pp.77-104. UCI : G704-SER000010583.2013.22..002

[13] Park Hyesook, The Development and Validation of a Multicultural Receptivity Scale for Korean College Students, Journal of Educational Studies, (2013), Vol.44, No.2, pp.219-248. UCI : G704-001618.2013.44.2.005

[14] J. D. Corrigan, L. D. Schmidt, Development and validation of revisions in the Counselor Rating Form, Journal of Counseling Psychology, (1983), Vol.30, No.1, pp.64-75. DOI: 10.1037/0022-0167.30.1.64

[15] Park Sookhyun, Factors Affecting Social Work Undergraduate Student's Attitude on Social Distance to Multicultural Groups, Honam University, Master's thesis, (2011)

[16] Kim Hyesook, Oh Seungseop, The stereotypes, affects and attitudes of college students and citizens toward North Koreans : A comparison with those toward other foreigners, Psychological Science, (1999), Vol.8, No.1. pp.1-12. 
[17] Nam Boyoung, Hong Ijun, Attitudes toward Multiculturalism: The Roles of Direct and Indirect Contact and their Interaction Effects, Social Science Research, (2021), Vol.37, No.3, pp.181-208. DOI: 10.18859/ssrr.2021.8.37.3.181

[18] Noh Yoonkoo, Lee OiSun, Influence of Multicultural contact Experience on the Relationship between Social Distance and Multicultural Acceptance among Nursing Student, Journal of Digital Convergence, (2018), Vol.16, No.1, pp.187195. DOI : $10.14400 / J D C .2018 .16 .1 .187$

[19] Bae Eunjoo, Jang Sohyun, The Effects of direct or Indirect Contact Experiences with Foreigners on citizen's Multicultural Acceptability -Mediation Effect of Threat Awareness-, Studies on Life and Culture, (2020), Vol.55, pp.5177.

[20] Sim Miyoung, Lee Onyu, An Analysis of Structural Relation of Multi-cultural Experience, Self-worth, Multi-cultural Stereotype and Multi-cultural Acceptance of Youth, The Journal of Humanities and Social science (HSS21), (2017), Vol.8, No.6, pp.547-560. DOI : 10.22143/HSS21.8.6.37 